\title{
DUAL FEED RF GUN DESIGN FOR THE LCLS*
}

\author{
L. Xiao, R.F. Boyce, D.H. Dowell, Z. Li, C. Limborg-Deprey, J. F. Schmerge \\ SLAC, Menlo Park, CA94025, U.S.A
}

\begin{abstract}
In order to remove the dipole field introduced by the coupler in existing S-band BNL/SLAC/UCLA 1.6 cell RF gun, a dual feed design for the LCLS RF gun is proposed together with several significant changes. The improvements include adopting $\mathrm{z}$-coupling instead of $\theta$ coupling, modifying the iris dimensions and profile to increase 0 - and $\pi$-mode separation from 3.4 to $15 \mathrm{MHz}$ and reduce the surface field on the iris, incorporating racetrack cavity shape to minimize the quadrupole field, increasing cooling for operation at $120 \mathrm{~Hz}$ and other small changes to improve performance and diagnostic capabilities. The 3D gun structure had been modelled with the parallel finite element complex eigensolver Omega3p to provide the desired RF parameters and to generate the gun cavity dimensions needed for fabrication. In this paper the RF gun design will be presented
\end{abstract}

\section{INTRODUCTION}

The RF gun for the Linac Coherent Light Source (LCLS) is based on the standard BNL/SLAC/UCLA 1.6 cell S-band RF gun [1]. The motivation of having dual feeds instead of a single feed is to eliminate the dipole field by symmetry. In the BNL/SLAC/UCLA design, the dipole field due to the single feed is compensated with another symmetrical opening used as a pump-out port opposite the power feed. In addition, the RF is coupled to the cavity using $\theta$-coupling instead of $\mathrm{z}$-coupling, which is also helpful in reducing the dipole field. However, even with the use of symmetric port compensation and $\theta$ coupling, there remains a dipole kick due to the power flowing into the cavity from a single feed. This shortcoming is overcome with the dual feed design. In this paper the racetrack cell shape is introduced to minimize the quadrupole moment $\gamma \beta \mathrm{r}$ from $4.4 \mathrm{e}-3 / \mathrm{mm}$ to $8 \mathrm{e}-5 / \mathrm{mm}$.

\section{LCLS RF GUN DESIGN}

The LCLS RF gun is required to operate at the $120 \mathrm{~Hz}$ linac repetition rate and with a $3 \mu$ s long $\mathrm{RF}$ pulse producing an electric field of $120 \mathrm{MV} / \mathrm{m}$ on the cathode plate. Under these conditions, the temperature rise at the iris of the coupling port in the prototype gun would have been $150^{\circ} \mathrm{C}$, subjecting it to thermo-mechanical fatigue. To reduce the pulsed heating, the coupling port is redesigned to produce the desired coupling coefficient but with acceptable temperature rise. In addition, z-coupling

*Work supported by the US.DOE under contract DE-AC02-76SF00515 "liling@ slac.stanford.edu instead of $\theta$-coupling is adopted to simplify fabrication and further reduce the pulsed heating [2].

In the standard RF gun the mode separation between 0 - and $\pi$-mode is $3.4 \mathrm{MHz}$. The amplitude of 0 -mode in the half cell is about $10 \%$ that of the $\pi$ mode when steady state is reached [3]. By increasing the mode separation to $15 \mathrm{MHz}$, this value can be reduced to less than $3 \%$. In addition, modifying the disk shape from circular to elliptical can reduce the peak surface field there from $11 \%$ higher to $2 \%$ lower than the field on the cathode with the shunt impedance maintained at the same value. A 3D computer model of the dual feed 1.6 cell RF gun with the modifications described is shown in Figure 1.

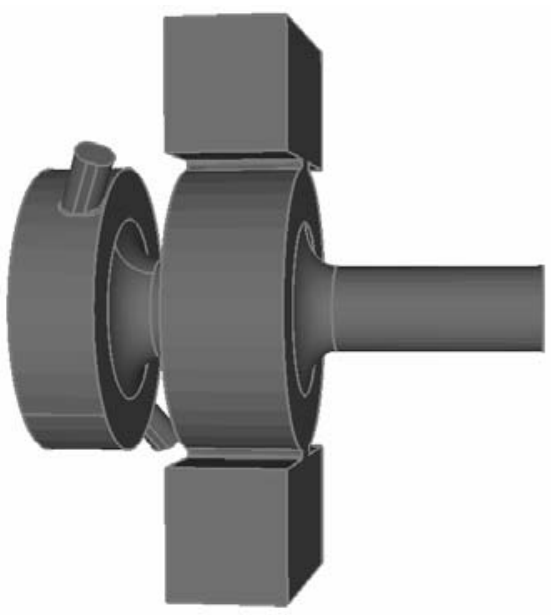

Fig. 1: 3D computer model of the dual feed RF gun for the LCLS

\section{NUMERICAL SIMULATIONS}

The finite element field solvers Omega2 and Omega3P were used to design the LCLS RF gun. Developed at SLAC, Omega2 is a serial 2D code while Omega3P is a 3D parallel code. These codes aim at high accuracy modelling of complex RF cavities and had been instrumental in the successfully development of the Damped, Detuned Structure for the NLC [4].

The LCLS RF gun operates in the $\pi$ mode with $\mathrm{f}=2.856 \mathrm{GHz}$. The RF properties were optimized first with Omega2. The 2D structure is shown in Figure 2 providing a $\pi$ mode frequency at $2.856 \mathrm{GHz}$ with nearly 
perfect field balance between the two cells of the gun cavity. The 0 - and $\pi$-mode separation is found to be $15 \mathrm{MHz}$ as desired.

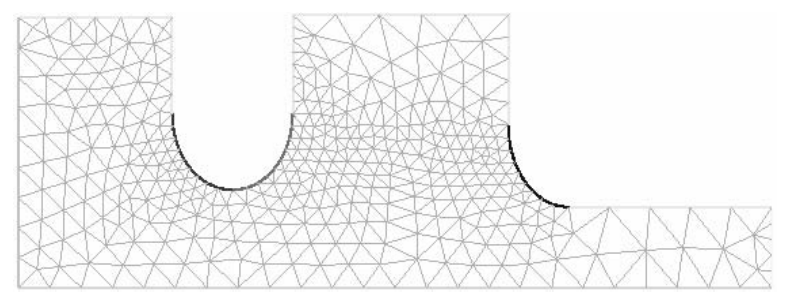

Fig. 2: The 2D mesh of the LCLS gun cavity as modelled by Omega2.

Based on the 2D cavity shape determined by Omega2, Omega3P was used to model the 3D gun structure that includes the input couplers as well as the laser ports. The input couplers were considered first so only a quarter model was needed in the simulation (see Figure 3). The boundary condition at the end of the waveguide was set to be matched. The complex eigensolver in Omega3p then was able to calculate the resonant frequency $f$, quality factor $Q_{0}$ and the external $Q_{\text {ext }}$. Because the dual feed couplers changed the cavity frequency, the cavity dimensions had to be readjusted to obtain the nominal RF parameters. .

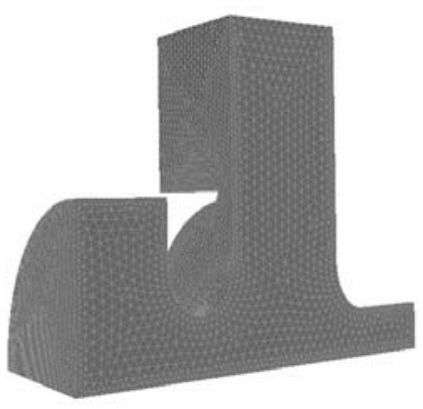

Fig. 3: A quarter model of the RF gun structure.

\section{PULSED HEATING}

In the single feed RF gun design, $\theta$-coupling was adopted to reduce the dipole field. At $120 \mathrm{~Hz}$ operation, the temperature rise at the end of the coupling aperture where it is curved could reach $150^{\circ} \mathrm{C}$. This pulsed heating will seriously limit the gun's life time [5]. Based on the NLC experience, the temperature rise due to the pulsed heating should be below $50^{\circ} \mathrm{C}$. A straightforward way to reduce the heating is to increase the radius on the inside surface of the coupler aperture. However, this rounding of the radius is difficult to machine with $\theta$ coupling so z-coupling is used instead. With z-coupling, because the iris has straight sides which extend the full length of the cell, the required radius can easily be fabricated.

In the z-coupling scheme, the width of the coupling slot and the rounding radius were adjusted to obtain a coupling coefficient around 2 and a temperature rise below $50^{\circ} \mathrm{C}$. The following equations were used to evaluate the temperature rise at the end of a RF pulse [6].

$$
\begin{aligned}
& \Delta T_{\max }=\frac{R_{s}}{K} \sqrt{\frac{D}{\pi}} \frac{1}{2} \int_{0}^{t_{p}}\left|H_{s \max }(t)\right|^{2} \frac{d t}{\sqrt{t_{p}-t}} \\
& R_{s}=\sqrt{\frac{\omega \mu}{2 \sigma}}=\frac{1}{\sigma \delta_{s}}, \quad \delta_{s}=\sqrt{\frac{2}{\omega \mu \sigma}} \\
& K=360 \mathrm{~W} / \mathrm{m} /{ }^{0} \mathrm{C} \quad \text { thermal conductivity } \\
& D=1.132 * 10^{-4} \mathrm{~m}^{2} / \mathrm{sec} \quad \text { specific heat }
\end{aligned}
$$

Here, $H_{\text {smax }}$ refers to the maximum surface magnetic field along the coupling slot on the inside of the cell and will decrease as the rounding radius $r 2$ increases. Figure 4 shows the results calculated assuming that the maximum electric field on the cathode is $120 \mathrm{MV} / \mathrm{m}$, the coupling coefficient is 2 and the pulse length $t_{p}$ is $3 \mu \mathrm{s}$. In the final design, the iris rounding $r 1$ and $r 2$ were determined to be $0.56 \mathrm{~mm}$ and $2.4 \mathrm{~mm}$ respectively, and the width of the z-coupling slot to be $13.2 \mathrm{~mm}$.

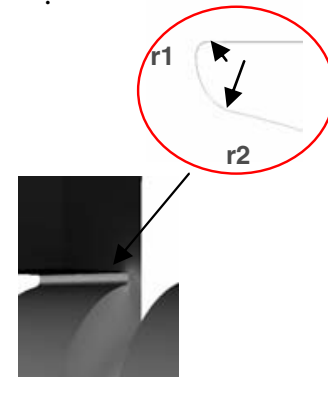

(a)

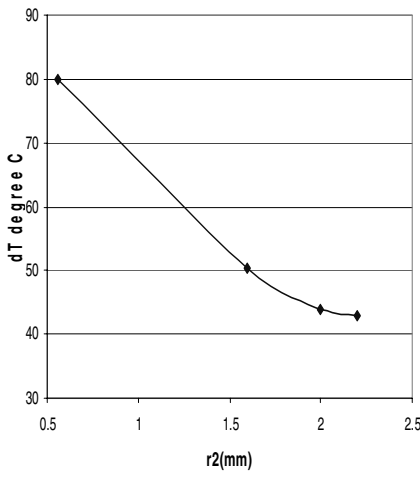

(b)
Fig. 4: (a) Surface magnetic field distribution;

(b) Temperature rise vs. rounding radius $\mathrm{r} 2$.

\section{QUADRUPOLE MOMENT}

While the dipole field is removed by the dual feed design, the quadrupole component remains unaffected so a racetrack shape has been adopted for the coupler cell to reduce its effect as shown in Figure 5a. In this geometry, the centre offsets of the two circles were adjusted to minimize the quadrupole field on the beam axis. This has led to a reduction of the maximum quadrupole moment $\gamma \beta \mathrm{r}$ from $4.4 \mathrm{e}-3 / \mathrm{mm}$ to $8 \mathrm{e}-5 / \mathrm{mm}$. Field determination to 
this level of accuracy was only possible by using $4^{\text {th }}$ order basis functions in Omega3P. Field maps generated with Omega3P were used in beam dynamics calculations of the gun emittance [3].

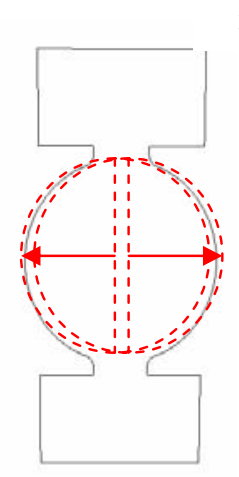

(a)

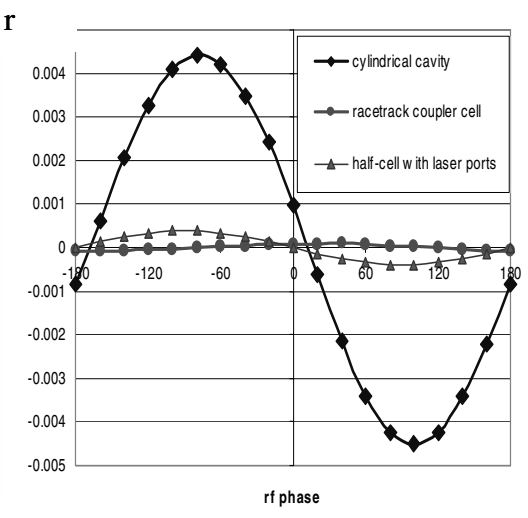

(b)
Fig. 5: (a) Racetrack coupler cell; (b) Quadrupole moment in the gun cavity.

\section{LASER PORT EFFECT}

The two laser ports in the half cell are located 45 degrees from the horizontal plane and admit the laser beam through an elliptical aperture. After adding the laser ports, the radius of the half cell was adjusted again to get the correct operating frequency and field balance. The maximum temperature rise at the laser port is $36^{\circ} \mathrm{C}$. The skew quadrupole moment in the half cell introduced by the laser ports was found to be about $3.85 \mathrm{e}-4 / \mathrm{mm}$ (see Figure 5(b)) which, as shown from PARMELA simulations, slightly changed the tuning but did not degrade either the slice or the projected emmittance [3].

\section{RF GUN PARAMETERS}

The final LCLS RF gun parameters are shown in Table 1.

Table 1: LCLS RF Gun parameters

\begin{tabular}{|l|l|}
\hline RF Parameters & Values \\
\hline F0 $(\mathrm{GHz})$ & $\mathbf{2 . 8 5 6 0 2 1}$ \\
\hline Q0 & $\mathbf{1 4 0 2 2}$ \\
\hline$\beta(=\mathrm{Q} 0 /$ Qext $)$ & $\mathbf{2 . 0}$ \\
\hline Mode Sep. $\Delta \mathrm{f}(\mathrm{MHz})$ & $\mathbf{1 5}$ \\
\hline$\Delta \mathrm{T} \max \left({ }^{\circ} \mathrm{C}\right)$ & $\mathbf{4 4}$ \\
\hline E0:E1 & $\mathbf{0 . 9 9 7 : 1}$ \\
\hline
\end{tabular}

The normalized longitudinal electric field along zaxis is shown in Figure 6.

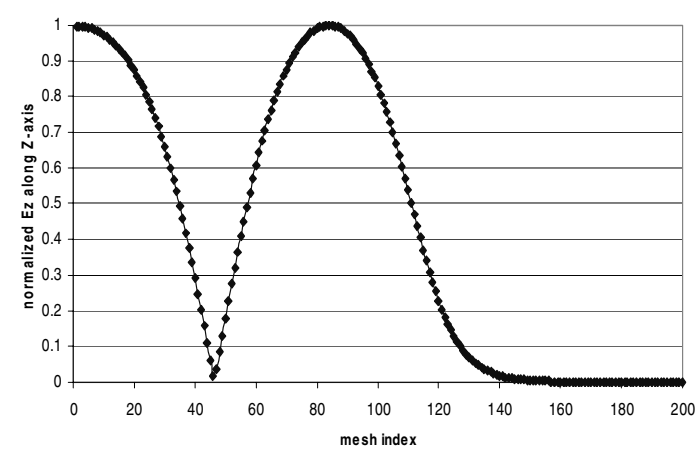

Fig. 6: On axis field profile in the LCLS RF gun cavity.

\section{SUMMARY}

The dual feed LCLS RF gun design is complete. The dipole mode is eliminated by the dual feed couplers. The quadrupole moment introduced by the couplers is greatly minimized using a racetrack cell shape. The maximum temperature rise due to the pulsed heating is reduced to below $50^{\circ} \mathrm{C}$ around the $\mathrm{z}$-coupling slot with larger rounding on the inside of cell. The complete thermal analysis of the average power dissipation for the new gun design is under way.

\section{ACKNOWLEDGEMENTS}

We appreciate the help from Kwok Ko throughout this work. We are also grateful to Liequan Lee for support on running Omega3P code.

\section{REFERENCES}

[1] D. T. Palmer et al., "Microwave Measurements of the BNL/SLAC/UCLA 1.6 cell Photocathode RF Gun", SLAC-PUB-95-6799

[2] J. Wang et al., "Report of the LCLS Injector RF Technical Review Committee", SLAC Memorandum, Nov. 22, 2004.

[3] C. Limborg-Deprey et al., "RF Design of the LCLS Gun”, LCLS-TN-05-3, Feb. 2005

[4] Z. Li et al., "High Performance Computing in Accelerating Structure Design and Analysis", ICAP2004

[5] R. Boyce et al., "Design Considerations for the LCLS RF Gun”, LCLS TN-04-4, April 2004

[6] D. P. Pritzkau et al., "Experimental Study of RF Pulsed Heating on Oxygen Free Electric Copper", Physical Review Spectial Topics - Accelerators and Beam, Vol.5, 112002 (2002) 